\title{
The Effect and Evaluation of Video Intervention and Education on Stress and the Effect of Operation of Patients Undergoing Oral Maxillofacial Operation
}

\author{
Tao Ran*, Zhang Yifang, Huang Minfeng, Li Jianan \\ The Stomatology College, The Public Health College, Harbin Medical University, Harbin, China
}

Email address:

376045883@qq.com (Tao Ran)

${ }^{*}$ Corresponding author

\section{To cite this article:}

Tao Ran, Zhang Yifang, Huang Minfeng, Li Jianan. The Effect and Evaluation of Video Intervention and Education on Stress and the Effect of Operation of Patients Undergoing Oral Maxillofacial Operation. Science Discovery. Vol. 7, No. 2, 2019, pp. 82-86.

doi: $10.11648 /$ j.sd.20190702.15

Received: April 10, 2019; Accepted: May 20, 2019; Published: May 23, 2019

\begin{abstract}
Objective: To evaluate the effect of video intervention and education on anxiety and pressure of patients undergoing oral maxillofacial operation. Method: a total of 96 patients were randomly divided into the experimental and control group, with 48 patients in each group. The control group were given routine intervention and education while the experimental group were given video intervention and education.The Zung Self-Rating Anxiety scale (SAS) and the Zung Self-Rating Anxiety scale (SDS) were used to evaluate the anxiety and depression degree of all patients one day before operation.The Visual Analogue Scale (VAS) and Dental visit satisfaction scale (DVSS) were used to assess the pain and satisfaction of all patients after operation one day after operation. Results: The SAS score of two group at 1 day before operation is (the control group: 11.52 \pm 1.93 , the experimental group: 11.66 \pm 2.01 ). There is no statistical significance between two groups. The SDS score of two group at 1 day before operation is (the control group:13.78 \pm 2.92 , the experimental group:13.89 \pm 3.02 ). There is no statistical significance between two groups. After video intervention and education, The SAS score of two groups is (the control group:11.36 \pm 1.90 , the experimental group: 7.85 \pm 1.00 ), The SDS score of two groups is (the control group:13.10 \pm 2.85 , the experimental group:8.46 \pm 1.51 ), there is significant difference between results of two groups $(p<0.05)$; the twice SAS and SDS score of the control group before one day of operation show have no statistical difference. The VAS score of two group at 1 day after operation is (the control group: $6.28 \pm 1.13$, the experimental group: $3.86 \pm 0.78)$. There is statistical significance between two groups $(p<0.05$ ). The DVSS score of two group after operation is (the control group: $70 \%$, the experimental group: $91 \%)$. There is statistical significance between two groups $(p<0.05)$. Conclusion: The video intervention and education can effectively relieve pressure of patients undergoing oral maxillofacial operation. Furthermore, it can also improve the effect of operation.
\end{abstract}

Keywords: Video Intervention and Education Before Operation, Operation of Oral Maxillofacial Surgery, Stress, Effect of Operation

\section{术前视频宣教对口腔领面外科手术患者心理压力 及手术效果的影响分析}

陶然", 张一方, 黄敏峰, 李佳楠

哈尔滨医科大学公共卫生学院口腔医学院, 哈尔滨, 中国

邮箱

376045883@qq.com(陶然) 
摘要：目的：研究术前视频宣教对口腔领面外科手术患者心理压力以及手术效果的影响。方法: 96例患者随机分为对 照组和试验组各 48 人, 对照组采用常规术前宣教, 实验组在常规宣教的基础上采用视频宣教, 术前一天采用焦虑自评 量表 (SAS评分) 和抑郁自评量表 (SDS评分) 评定患者的心理压力状态, 术后一天采用疼痛视觉模拟量表(VAS)和 口腔治疗满意度量表（DVSS）测评患者术中的疼痛程度和口腔治疗满意度。结果：术前一日两组患者的SAS、SDS 评分 (对照组: 13.78 2.92 , 实验组: 13.89 \pm 3.02 ), 差异无统计学意义; 经视频宣教后, SAS 评分 (对照组: 11.36 \pm 1.90 , 实验组: 7.85 1.00$)$, SDS评分 (对照组: $13.1 \pm 2.85$, 实验组: $8.46 \pm 1.51$ ), 差异有统计学意义 $(P<$ $0.05)$; 对照组术前两次调查评分差异无统计学意义。术后一日两组患者的疼痛状况（对照组： $6.28 \pm 1.13$, 实验组: $3.86 \pm 0.78)$, 差异有统计学意义 $(P<0.05)$; 口腔治疗满意度实验组高于对照组 $(91 \% / 70 \%)$, 差异有统计学意义 $(P<$ 0.05)。结论: 利用视频进行术前宣教, 能有效缓解患者心理压力, 提高手术治疗效果。

关键词: 术前视频宣教, 口腔领面外科手术, 心理压力, 手术效果

\section{1. 引言}

口腔领面外科手术是治疗口腔领面部各种良恶性肿 瘤、修复组织缺损、腺体吻合、关节成形、淋巴结清 扫、畸形矫正的主要手段 [1-2]。因为口腔领面外科手术 的特殊性, 临床研究显示, 口腔领面外科术后患者口腔 粘膜溃疡、经常出现吞咽障碍、创口出血、感染等并发 症发生率较高, 重者合并交流不畅以及面部形态改变 等, 术后患者会产生紧张焦虑、恐惧抑郁等不良情绪心 理作用, 影响医生手术治疗和患者术后康复 [1-9]。有效 的口腔领面外科手术前宣教可以提高患者对疾病及手术 的认知, 减轻患者的心理压力, 改善患者焦虑抑郁的状 况, 提升患者手术信心更好地配合医生进行手术治疗 [1012]。传统的术前宣教多为口头或书面式宣教, 内容形式 单一, 十分抽象, 不利于患者及家属的接受理解, 其效 果相对有限, 而视频式宣教能够有效加深患者对手术相 关知识的理解掌握 [13]。

目前, 术前视频宣教对临床外科患者心理压力及手 术效果的影响的报道有数例, 均认为多媒体、多元化的 术前宣教能有效减轻患者心理压力, 提高术前宣教依从 性, 提升手术效果[14-16], 但是目前与口腔领面外科有 关的只有对牙槽外科及口腔种植手术患者的研究, 这些 研究发现通过多媒体宣教干预, 展示教学图片、模型、 播放幻灯片等形式进行宣教, 能有效缓解口腔种植手术 患者术前的焦虑心理, 减轻术中疼痛[17、23-27]。但口 腔领面外科手术相比牙槽外科及种植手术，手术范围和 创伤更大，患者的心理压力也相应更大。目前仍没有关 于该方面的研究报道。本研究选取本院96例接受口腔领 面外科手术治疗的患者, 应用自制的术前宣教视频, 调 查研究术前视频宣教对口腔领面外科患者心理状况及手 术效果的影响, 探讨视频宣教的有效性并为其推广应用 提供理论依据。

\section{2. 资料与方法}

\section{1. 一般资料}

选取2017年6月至2019年2月在本院口外科接受治疗 的96例患者作为研究对象。纳入标准: 年龄为 18 周岁以 上的成年人, 意识清楚, 与调查人员沟通无障碍, 自愿
合作填写调查表内容（包括既往史和用药史）; 说明研 究目的后, 愿意参加本研究者。排除标准: 有抑郁、焦 虑病史者、精神分裂症患者; 一个月内服用过抗焦虑或 镇静药者; 不愿意参加本研究者。

\section{2. 实验分组与干预方法}

将上述符合纳入排除标准并签署知情同意的患者随 机分配到对照组和实验组。对照组由医护人员做常规的 口头或书面宣教, 告知病情、手术方法、术前禁忌、术 中和术后可能出现的不适情况及相应的处理办法。实验 组在此基础上增加由本研究特别拍摄的口腔外科手术宣 教视频, 做更为形象的宣教, 将手术室的环境、手术流 程和麻醉效果等更加生动清晰的展现给患者, 所选择的 多媒体资料避免出现引起或加重患者反感恐惧的图片, 旨在疏导患者心理, 使其放松、消除顾虑, 引导其更加 主动的配合手术。

\section{3. 观察指标}

\subsection{1. 心理状态评估}

采用焦虑自评量表（SAS）[16-18]和抑郁自评量表 (SDS) 进行评估[16-18]。这两种自评量表由William W.K. Zung编制。我国学者已将其翻译为中文使用, 并有 良好的信度和效度 [19]。

焦虑状况评估 采用焦虑自评量表（SAS）进行评 估。该量表有 20 个条目, 其中 10 个为正向评分, 10 个为 反向评分。按Likert4级评分制。1分：没有或很少时间有 （过去一周内, 出现这类情况的日子不超过一天）；2 分: 小部分时间有（过去一周内, 有 1-2天有过这类情 况）; 3 分：相当多时间（过去一周内，3-4天有过这类 情况）；4分：绝大部分或全部时间（过去一周内, 有57 天有过这类情况）。标准分数越高焦虑程度越严重。

抑郁状况评估 采用抑郁自评量表 (SDS) 进行评 估。该量表有 20 个条目, 为Likert4级评分, 分值范围为 1 4。每个分数代表意义与SAS相同。同样, 标准分数 越高抑郁程度越严重。

\subsection{2. 疼痛程度评估}

采用疼痛视觉模拟量表 (Visual Analogue Scale, VAS)[20]测评患者术中的疼痛程度。设计为一条长约 10 
$\mathrm{cm}$ 的直线, 0 分表示无痛, 10 分表示剧烈难于忍受的疼 痛。根据患者的感受程度，在直线上相应部位作记号， 记录疼痛分值, 分值越高说明疼痛程度越高。

\subsection{3. 口腔治疗满意度评估}

在术后对两组患者就手术治疗满意程度进行调查, 参照了 Norman L.Corah 等研制的牙科满意度量表 (Dentalvisit satisfactionscale,DVSS）[16、21]设计问卷， 共有 10 项针对医生问题, 主要内容有:信息获得与沟通 (包括通过医生讲解和沟通后, 对自己目前、未来口腔 状况的了解, 医生向我讲解清楚), 相互理解接受 (我 感到医生很理解我的: 治疗要求、对于疼痛产生的不安 情绪, 医生在治疗过程中对我平等相待），医疗技术水 平（医生在治疗过程中是否认真、是否粗暴，我对医生 的治疗是否满意, 医生是否清楚每一步该如何进行) 等。调查表采用Likert5级评分制, 分值范围为 $1 \sim 5$ 分。 分值越高表明患者对本次口腔外科治疗的满意度越高。

\section{4. 研究的方法}

两组患者于未行手术宣教前填写: 一般资料调查 表、焦虑自评量表（SAS）和抑郁自评量表（SDS），之 后两组患者分别按不同的干预方法进行手术前宣教, 宣 教后手术前再次填写SAS、SDS量表, 手术完成后对患者 进行疼痛程度(VAS)、牙科满意度(DVSS)测评。

\section{5. 统计学处理}

应用SPSS22.0统计软件进行统计学处理。计量资料 用 $\bar{x} \pm \mathrm{S}$ 表示, 采用 $\mathrm{t}$ 检验, 计数资料用 $\chi^{2}$ 检验, 以 $P<0.05$ 为差异有统计学意义。

\section{3. 结果}

\section{1. 两组研究对象一般资料比较}

本研究共调查 96 人, 以随机数字表法将患者分为对 照组和实验组各 48 例, 其中对照组男性 26 人, 女性 22 人; 实验组男性 25 人, 女性 23 人。两组间一般资料比 较, 差异无统计学意义 $(P>0.05)$, 具有可比性（表 1)。

表1 两组研究对象一般资料比较 $(\mathrm{n}=48)$ 。

\begin{tabular}{lll}
\hline & 对照组 & 实验组 \\
\hline 男性 (例) & 26 & 25 \\
女性 (例) & 22 & 23 \\
年龄 (岁) & $45.18 \pm 10.11$ & $45.51 \pm 9.30$ \\
口外手术方式: & & \\
阻生齿拔除术 & 9 & 10 \\
残根拔除术 & 12 & 13 \\
腮腺肿物摘除术 & 8 & 10 \\
唇部肿物摘除术 & 15 & 14 \\
其它 & 2 & 3 \\
受教育方式 (例) : & & \\
初中 & 9 & 10 \\
高中 & 18 & 19 \\
大学及以上 & 20 & 20 \\
\hline
\end{tabular}

\section{2. 术前心理压力的评估结果}

宣教前, 两组患者焦虑值 (SAS) 评分、抑郁值 (SDS) 评分均较高, 经过术前视频宣教后, 实验组的 焦虑状况、抑郁状况有所缓解, 与对照组相比, 差异有 统计学意义 $(P<0.05) \quad($ 表 2$)$

表2 两组研究对象心理状况及疼痛程度评分比较。

\begin{tabular}{|c|c|c|c|c|c|c|}
\hline & 视频宣教前 & & & 视频宣教后 & & \\
\hline 项目 & 对照组 & 实验组 & $\mathbf{P}$ & 对照组 & 实验组 & $\mathbf{P}$ \\
\hline 焦虑值（SAS） & $11.52 \pm 1.93$ & $11.66 \pm 2.01$ & $>0.05$ & $11.36 \pm 1.90$ & $7.85 \pm 1.00$ & $<0.05$ \\
\hline $\begin{array}{l}\text { 抑郁值（SDS） } \\
\text { 疼痛值（VAS） }\end{array}$ & $13.78 \pm 2.92$ & $13.89 \pm 3.02$ & $>0.05$ & $\begin{array}{l}13.1 \pm 2.85 \\
6.28 \pm 1.13\end{array}$ & $\begin{array}{l}8.46 \pm 1.51 \\
3.86 \pm 0.78\end{array}$ & $\begin{array}{l}<0.05 \\
<0.05\end{array}$ \\
\hline
\end{tabular}

\section{3. 术后疼痛评估结果}

术后疼痛的比较, 对照组 VAS值为 $6.28 \pm 1.13$, 实验 组 VAS 值为 $3.86 \pm 0.78$, 实验组评分小于对照组, 差异有 统计学意义 $(P<0.05)$ 。(见表2)

\section{4. 手术效果的评估结果}

手术效果的满意度, 实验组高于对照组, 差异有统 计学意义 $(P<0.05)$ 。（见表3）

表3 牙科满意度量表（DVSS）。

\begin{tabular}{lllll}
\hline 组别 $(\mathbf{n})$ & 信息交流 & 理解与宽容 & 技术水平 & 总评分 \\
\hline 实验组 $(48)$ & 14 & 18 & 13 & 45 \\
对照组 & 11 & 10 & 11 & 93.75 \\
\hline
\end{tabular}

\section{4. 讨论}

国内外研究表明口腔科患者治疗前容易出现焦虑的 症状[2、5], 这是由于许多患者对口腔外科疾病的认知能 力有限、担心手术效果及预后等, 产生的紧张焦虑、恐
惧抑郁等负性情绪。这些负性情绪通过神经内分泌调节 的变化造成患者机体生理功能紊乱、免疫力下降, 进而 影响手术的顺利进行及患者术后康复[3-9、12、22]。而 术前宣教可以通过向患者普及手术有关的基本知识、手 术流程、预期效果等提高患者的认知能力和安全感, 有 效减轻患者心理压力[17]。 
本研究将视频术前宣教与常规术前宣教进行对比, 研究表明采用视频宣教的实验组无论是焦虑状况还是抑 郁状况都明显好于采用常规宣教的对照组, 心理压力得 到了明显的改善。这是因为传统的口头或书面式术前宣 教, 形式呆板, 内容枯燥, 示范抽象, 不利于患者及家 属的理解接受, 此外还受护理人员语言表达能力的影 响, 宣教效果差异较大。而视频式宣教直观性强、图文 声像并茂, 可将复杂抽象的内容通过视频形象地展示出 来, 有助于患者的理解掌握。通过视频中对我院口腔领 面外科手术室真实情景的再现, 让患者更多的了解手术 流程步骤, 减轻患者因自身主观猜测所导致的焦虑、畏 惧心理, 从而缓解其心理压力, 更好地配合医生进行治 疗。视频式宣教还可确保宣教内容的规范化、标准化、 统一化, 降低护理人员的工作量, 提高工作效率。

疼痛作为一种心理现象有明显的主观性, 心理因素 会影响疼痛的感知, 国内外研究表明口腔手术前的焦虑 水平与术后疼痛程度显著相关, 焦虑抑郁的情绪会降低 患者的痛阈引起疼痛[16-20]。本研究显示采用视频宣教 的患者术后疼痛程度评分明显低于对照宣教组, 表明视 频宣教形式有效地缓解了患者的疼痛。这可能是由于视 频宣教后患者焦虑抑郁情绪得到了改善, 对手术的心理 负担减轻、对手术操作有更好的适应能力, 从而在一定 程度上减轻了对疼痛的感知程度。

根据表 3 , 视频宣教组的DVSS总评分较高, 表明对 手术效果的总体满意程度较高。在信息交流、理解与宽 容、技术水平三个方面视频宣教组也均优于常规宣教 组, 其中满意度提高最为显著的是理解与宽容方面。视 频宣教可最大程度地提供多种信息, 患者可重复观看, 通过视频的直观展示和相关讲解, 提高了患者对宣教内 容的理解掌握。在视频宣教的过程中还加强了医生与患 者之间的沟通, 有助于相互了解、使患者感觉受到更多 重视与人文关怀, 提高了患者对医生的信任程度。此外 视频带来的画面感与单纯的口头宣教相比, 患者更容易 理解接受, 更容易遵循医生嘱托的手术禁忌和注意事 项, 保证手术的顺利进行。

总之, 通过本研究发现视频术前宣教与常规术前宣 教相比, 不仅可以提高患者对口腔领面外科手术相关知 识的认知、缓解患者的心理压力, 还可以减轻患者对手 术疼痛感受、提高对手术效果的满意程度。在此过程中 还加强了医生与患者之间的沟通交流, 促进了医患之间 相互信任, 有助于构建新时期和谐的医患关系。因此视 频宣教作为一项成本低廉、操作简单且患者易于理解接 受的方法, 值得在临床上推广应用, 为更多的患者服 务, 以期使更多的患者有更佳的就医体验。

\section{5. 结论}

口腔领面外科手术患者经术前视频宣教后, 对心理 压力及手术效果的影响, 从焦虑水平、抑郁水平、疼痛 程度评估, 均低于普遍人群; 对口腔治疗满意度的评价 高于普遍人群。一般人口学因素的性别、文化程度、年 龄不影响术前视频宣教对口腔领面外科手术患者的心理
压力及手术效果的评估。利用视频进行术前宣教, 能有 效缓解患者心理压力, 提高手术治疗效果。

\section{参考文献}

[1] 曾晓燕, 周维, 张波. 口腔领面部间隙感染的临床分析 [J]. 中华医院感染学杂志, 2015(2): 421-423。

[2] 史俊南. 现代口腔内科学 [J]. 北京: 高等教育出版 社.2000:359。

[3] 陈婷. 对口腔领面外科手术患者实施优质护理满意度及负 性心理情绪影响的探讨 [J]. 世界最新医学信息文摘, 2016(2):174-175。

[4] 叶国凤, 邵丽红, 孙红燕, 等. 45例口腔领面部游离组织 瓣移植术并发症的观察及护理[J]. 中华护理杂志, 2014, 49( 11): 1352-1355。

[5] 黄倩,何立弘,巢永烈. 口腔焦虑症[J]. 国外医学口腔医学分册, 2003, 30( 5): 405-407。

[6] 徐立平, 许彪, 李曙晖, 等. 183例口腔内科患者牙科畏惧症 调查[J].华西口腔医学杂志, 1993, 11 (4): 296-297。

[7] Humphris GM, Dyer TA, Robinson PG. The modified dental anxiety scale:UK general public population norms in 2008 with further psychometrics and effects of age [J]. BMC Oral Health. 2009, 9: 20.

[8] Humphris G,King K. The prevalence of dental anxiety across previous distressing experiences [J]. J Anxiety Disord. 2011, 25 (2): 232-236.

[9] Nuttall NM, Bradnock G, White D, et al.Dental attendance in 1998 and implications for the future [J]. Br Dent J.2001, 190 (4): $177-82$.

[10] 陈冀刚. 口腔健康宣教对洁牙患者牙科焦虑心理的帮助 [J]．中国社区医师，2017,33(1) 136。

[11] 龚雪. 口腔健康宣教对促进牙周病治疗效果的临床研究 [J]．全科口腔医学杂志, 2018，32（5）：110-111。

[12] Alansari BM. Psychometric properti es of the Arabi cversion of the Dental Cognition Questionnaire [J]. Community Dent Health, 2006, 23 (2): 83-90.

[13] 张敬, 阎培菁. 移动式多媒体在术前宣教中的应用与效果 评价[J].实用临床护理学杂志, 2018,3(9):192-193。

[14] 张庆芬, 赵岳, 李之华.多媒体健康教育对胰腺癌化疗患 者家属疾病不确定感、焦虑及应对的影响 [J].护士进修杂 志, 2018，33(2): 171-174。

[15] 冯硕, 豆艳明.多媒体健康宣教对冠状动脉搭桥术患者术 前焦虑的效果评价 [J]. 护理实践与研究, 2016, 13(6): 149-150。

[16] 杨梅,余娜,田聪林.多元化术前宣教模式对减轻患者术前心 理压力及手术效果的影响分析 [J]. 检验医学与临 床,2016,13(16):2348-2350。 
[17] 孔宁华，吴迪，吴军楼等.多媒体宣教干预对口腔种植手 术患者牙科焦虑和疼痛的影响 $[\mathrm{J}]$. 口腔领面修复学杂志, 2018,19(6): 335-339。

[18] Greer JA,Traeger L,Bemis H,et al.A Pilot randomized controlled trial of brief cognitive-behavioral therapy for anxiety in patients with terminal cancer[J].Journal of Psychosomatic Research, 2012,17(10):1337-1345.

[19] 段泉泉, 胜利.焦虑及抑郁自评量表的临床效度[J].中国心 理卫生杂志，2012，26（09）：676-679.

[20] 陈香, 张长春, 邵 丹, 等.认知松弛训练对改善口腔种植 手术患者心理状况及术中疼痛的研究[J]. 中国临床护理, 2016，8(6): 465-468。

[21] Norman L. Corah, Robert M. O Shea,Lawrence F.Pace, and Sait K. Seyrek. Development of a Patient Measure of Satisfaction with the Dentist: The Dental Visit Satisfaction Scale. Journal of Behavioral Medicine, VoL7, NO.3, 1984: 367-373.
[22] Mansell W, Morris K. The Dental Cognitions Questionnaire in CBT for dental phobia in an adolescent with multiple phobias [J]. J Behav Ther Exp Psychiatry, 2003, 34 (1): 65-71.

[23] 王艳, 齐万华, 单连启, 等. 口腔门诊患者牙科焦虑症的 影响因素 [J]. 中国健康心理学杂志, 2014, 22(1): 2526 。

[24] 陈莺, 林丽婷, 赖娘妹, 等. 牙槽外科焦虑症的调查和分 析[J]. 中华口腔医学研究杂志: 电子版, 2013, 7(2): 122124 。

[25] 何天鹏, 李 昕. 综合医院牙科门诊老年患者焦虑症及影响 因素研究[J]. 中华老年口腔医学杂志, 2013, 11(3): 166169 。

[26] 陈阳, 刘敏. 口腔种植患者牙科焦虑症影响因素分析 [J].上 海口腔医学, 2016，25(6): 711-714。

[27] 张虹, 潘在兴, 黄颖. 重度骨量不足种植牙患者牙科焦虑症 分析及心理护理 [J]. 中国口腔种植学杂志, 2009, 14(3): 88-90。 